\title{
Mutants of Aspergillus nidulans deficient in nuclear migration during hyphal growth and conidiation
}

\author{
A. John Clutterbuck
}

Tel: +44 413398855 ext. 5105. Fax: +44 41330 5994.e-mail: GBGA15@vms.gla.ac.uk

Department of Genetics, Glasgow University, Church Street, Glasgow G11 5JS, UK

\begin{abstract}
Anucleate primary sterigmata (aps) mutants of Aspergillus nidulans are partially blocked in conidiation (asexual sporulation) due to failure of the organized migration of nuclei into the conidiophore metulae. The mutants also have a slightly reduced hyphal growth rate and irregular distribution of nuclei in vegetative hyphae; the hyphal phenotype appears somewhat more variable than the conidiation defect. The mutants fall into two complementation groups, apsA and apsB, mapping on chromosomes IV and VI, respectively. apsB mutants are also partially defective in sexual reproduction.
\end{abstract}

Keywords: Aspergillus nidulans, nuclear movement, conidiation, aps mutants

\section{INTRODUCTION}

A collection of mutants of Aspergillus nidulans defective in conidiation (Clutterbuck, 1969a) has led to intensive genetic and molecular studies of this process (reviewed by Timberlake, 1990; Clutterbuck \& Timberlake, 1992). The original collection included only mutants which were specifically defective in conidiation, and therefore normal for vegetative growth. In addition, it became apparent that in the mutants of greatest interest the conidial apparatus was abnormal in morphology, but was not restricted in growth.

One group of mutants are of interest, although they were excluded from the original study because they failed to meet both of the above criteria; these mutants produced conidiophores which were apparently normal up to the metula stage, but showed no further growth or development. Closer examination showed that the metulae (also known as primary sterigmata) were anucleate, and the mutants were given the designation aps, standing for 'anucleate primary sterigmata'. These mutants were described briefly by Clutterbuck (1977), but details of their phenotype are given here for the first time.

\section{METHODS}

Strains and media. Strains of $A$. nidulans and general methods have been described by Pontecorvo et al. (1953) and Clutterbuck (1974). Strain G051 from the Glasgow stocks, genotype biA1; ve $A 1$ (biotin-requiring, 'velvet' colony morphology, therefore able to conidiate in the dark; Mooney \& Yager, 1990) was used

Abbreviation: DAPI, diamidino-2-phenylindole. as morphological wild-type. Morphological studies were all made on direct derivatives of this strain obtained by mutation (Clutterbuck, 1969a). aps- 8 was obtained after nitrous acid treatment, $a p s-1$ to -7 and -9 to -23 after diethyl sulphate treatment, $a p s-34$ and -36 after treatment with ultraviolet light, and $a p s-26$ and -27 after $N$-methyl- $N^{\prime}$-nitro- $N$-nitrosoguanidine treatment. Minimal and complete media are described by Clutterbuck (1974); all studies of growth and morphology were made on cultures grown on complete agar medium at $37^{\circ} \mathrm{C}$.

Growth rate and conidiation. Colony growth rates were measured with a ruler on the back of the dish, assessing change in colony diameter between days 2 and 4 after point inoculation in the centres of four replicated dishes. Dish cultures for quantification of conidiation were inoculated with $10^{5}$ conidia per dish in a $4 \mathrm{ml}$ complete agar top layer on a bottom layer of the same medium.

Microscopy. Slide cultures for microscopic examination consisted of a film of complete medium on a microscope slide. Microscope slides for this purpose were sterilized and degreased by thorough flaming, and allowed to cool. Medium containing half-strength agar, was then poured over and allowed to drain off before it set. Slides were point-inoculated with conidia, and incubated at $37^{\circ} \mathrm{C}$ supported on glass rods over water in a Petri dish for $48 \mathrm{~h}$. Slides for examination of conidiophores were left uncovered, whilst those for examination of hyphae were covered with permeable cellophane and a further layer of medium as described by Clutterbuck \& Roper (1966). Slides were either fixed and stained for acridine orange fluorescence, as described by Clutterbuck \& Roper (1966), or for diamidino-2-phenylindole (DAPI) fluorescence according to Bergen \& Morris (1983). Cultures were examined using a Reichert fluorescence microscope or a Leitz epifluorescence/phase contrast microscope.

Statistical methods. Bailey (1959) was consulted for statistical methods. 

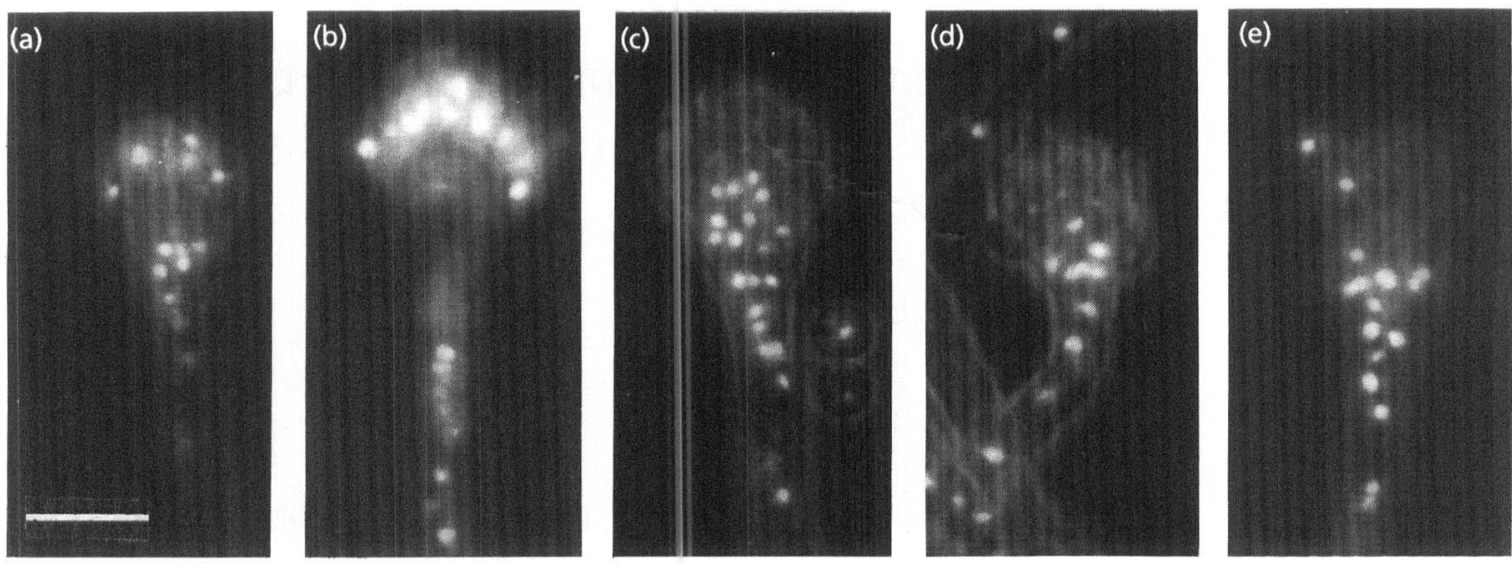

Fig. 1. DAPI-fluorescence of wild-type and apsA6 conidial heads. Colonies were grown on microscope slides, fixed and stained, and examined using combined epifluorescence and phase contrast. (a) and (b) Wild-type at stages at which the vesicles bear nucleated metulae. (c)-(e) apsA6 heads in which metulae have developed phialide buds but are still anucleate; (e) shows a conidial head in which one metula has acquired a nucleus and has developed a normal phialide. Bar, $10 \mu \mathrm{m}$.

\section{RESULTS}

\section{Complementation and mapping}

A total of 33 mutants with similar phenotypes, indicating a developmental block at the metula stage, were tested for complementation in heterokaryons with a limited number of tester strains carrying complementing auxotrophs. Nine mutants (aps-1, $-2,-5,-6,-9,-18,-27,-34$ and -36 ) fell into one clear group, members of which complemented with the aps-8 tester, but failed to complement with aps-6; these were designated as aps $A$ mutants. A second group of seven mutants (aps-8, -10,-14, -17, -19, -20 and -26) were designated $a p s B$ mutants, complementing aps $A 6$ but not aps-8. Cytological examination of all these mutants revealed the anucleate primary sterigmata phenotype, as described below, whilst the remaining mutants, although blocked at a similar developmental stage, did not show this specific defect. They had a variety of phenotypes, mostly less distinctive than that of those classified as aps mutants, and complementation tests implied that they represented a number of loci. They have not been studied further.

aps $A 6$ and $a p s B 8$ mutants are recessive in diploids, and the ability of mutants to complement one or other tester strain implies that all mutants are recessive in heterokaryons. Both loci have been mapped by standard methods (Pontecorvo et al., 1953; Clutterbuck, 1974): aps $A$ is 25 units distal to pyro $A 4$ on the right arm of chromosome IV, and aps $B$ is between nic $C$ and metbB, at distances of 2 and 11 map units, respectively, on the right arm of chromosome VI (Clutterbuck, 1974, 1993).

\section{The conidiophore phenotype}

In the wild-type, an average of 75 metulae (a number recalculated from data presented in Clutterbuck, 1969b) are budded from the upper surface of the conidiophore vesicle, and when these are approximately $3.8 \mu \mathrm{m}$ in length, one nucleus migrates into each bud. According to Mims et al. (1988), nuclear division takes place in the mouth of each bud, one daughter nucleus moving into the bud, the other remaining in the vesicle. In aps mutants, organized nuclear migration fails, and the majority of metulae remain anucleate (Fig. 1). The course of nuclear division in the vesicle is not easy to observe since massed nuclei tend to obscure each other, but it was clear that vesicles of aps mutants contained many nuclei at the time when migration into the metulae would normally occur, implying that mitosis had proceeded normally up to this stage.

The anucleate aps metulae grow to almost full size and produce phialide buds at their tips, but no further growth or development takes place. In all aps mutants examined, however, some metulae had acquired nuclei, and these metulae were able to develop phialides and conidia. A lawn of the wild-type strain began to conidiate at approximately $24 \mathrm{~h}$ after inoculation and developed $6.7 \times 10^{5}$ conidia $\mathrm{mm}^{-2}$ by 49 h. aps $A 6$ and apsB8 strains grown in parallel gave $1.4 \%$ and $1.8 \%$ of the wild-type yield, respectively, at $49 \mathrm{~h}$. Comparison of cultures at earlier stages suggested that conidiation on the small proportion of nucleated metulae proceeded at the same rate as in the wild-type. No obvious responses of the conidiophore phenotype to changes in the medium were detected, but all $a p s B$ mutants conidiated slightly better at a higher temperature $\left(42^{\circ} \mathrm{C}\right)$ than at 37 or $25^{\circ} \mathrm{C}$.

A proportion of the mutant conidia were somewhat enlarged and misshapen. Using fluorescence microscopy, $16 \%$ of apsB 8 and $5 \%$ of aps $A 6$ conidia were seen to be binucleate, whilst 200 wild-type conidia were all uninucleate. Germination at $6 \mathrm{~h}$ after inoculation for the conidia of four aps mutants, averaged $68 \%$ compared to $89 \%$ for the wild-type. No obvious abnormality of nuclear distribution was detected in mutant germlings. 


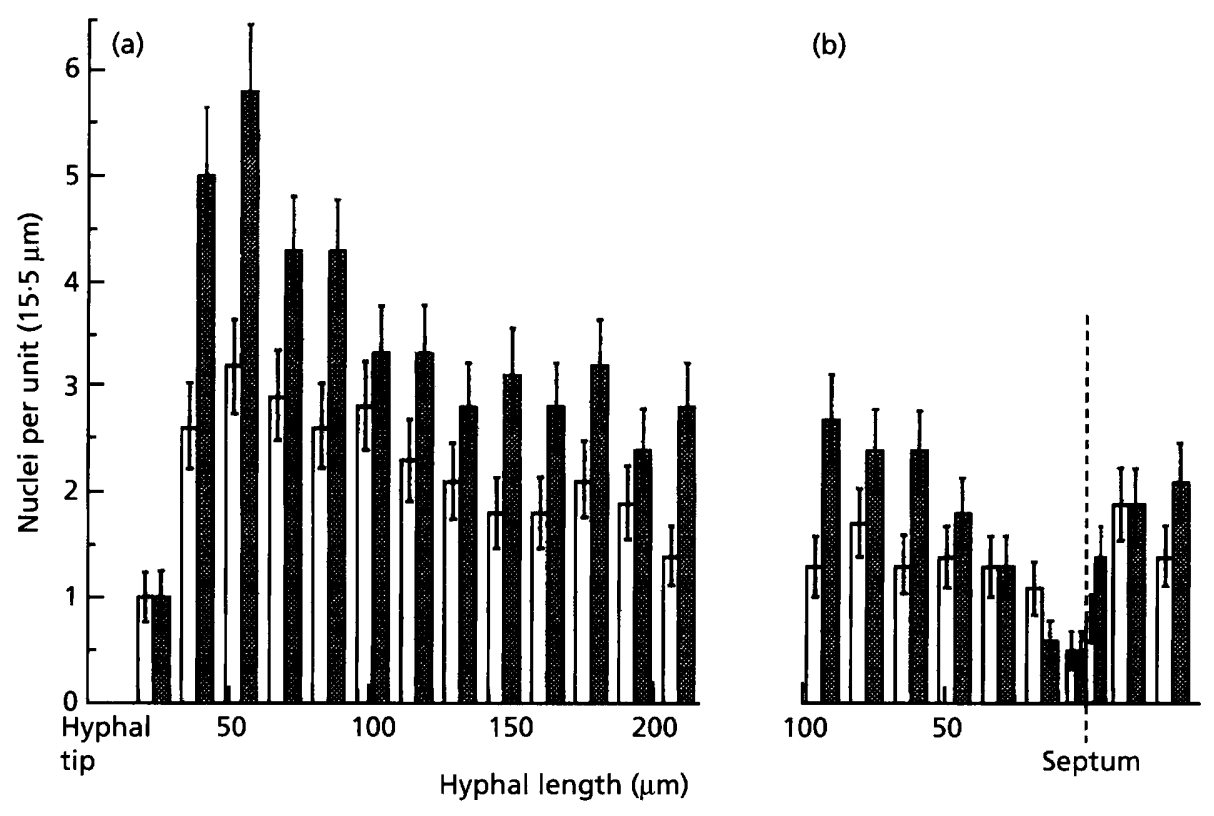

Fig. 2. Distribution of nuclei along the hyphal tip cells of wild-type and apsA6. Hyphae were grown on slides and examined by acridine orange fluorescence. The histogram bars represent the mean number of nuclei per unit length of hypha (micrometer eyepiece unit: $15.5 \mu \mathrm{m}$ ). Four hyphal tip cells from each of four slides of each strain were counted, giving a total length of $5812.5 \mu \mathrm{m}$ for the wild-type and $4913.5 \mu \mathrm{m}$ for apsA6. Open bars, wild-type; shaded bars, apsA6. (a) Hyphae aligned at their tips. (b) Hyphae aligned at the septum demarcating the rear of the tip cell. Nuclei in the unit including the septum are split according to their positions in front of, or behind the septum; the heights of the halfcolumns are adjusted $(\times 2)$ to take account of this. Error bars $=$ SEM.

Examination of old aps conidial heads suggested that many metulae eventually acquire nuclei, and formed swollen, multinucleate structures, but there was no evidence that these were able to develop conidia.

\section{Hyphal phenotype}

Colonies of most aps mutants on agar grew somewhat more slowly than the wild-type. Growth rates for mutants $a p s B 8,-A 18,-B 26$ and $-A 27$ were not significantly different from that of the wild-type, but the remaining mutants grew significantly more slowly, with growth rates ranging from $68 \%$ to $85 \%$ of the wild-type. Both the growth defect and differences in nuclear distribution detailed below were somewhat less pronounced on minimal salts medium than on complete medium. The latter was therefore used in all further studies. The morphology of vegetative cultures, including the hyphal branching pattern, appeared normal in aps mutants.

Hyphae of all aps mutants were examined by fluorescence microscopy. The mutants exhibited a spectrum of features, including high concentrations of nuclei in hyphal tip cells (Fig. 2) and distribution of nuclei in clusters separated by conspicuous gaps (Fig. 3). In a number of instances it was noticed that a cluster of nuclei distal to a septum coincided with a long gap in front of the septum. A few hyphae in most of the of aps strains examined also exhibited excessive variation in nuclear size, including the presence of giant nuclei. Some or all of these features were evident in all mutants with the exception of $a p s B 8$, which was cytologically indistinguishable from the wild-type.

To quantify nuclear distribution, slide cultures of a representative mutant (aps $A \sigma$ ) were compared with the wild-type. Four slides of each genotype were examined, selecting four hyphal tips at the periphery and following each for a distance of 40 units back into the colony, with the aid of an eyepiece micrometer delineating a unit length of $15.5 \mu \mathrm{m}$. Side branches were ignored. The mean length of the wild-type hyphal tip compartment was $409 \mu \mathrm{m}$, compared to $343 \mu \mathrm{m}$ for the aps $A$ strain, a difference which is significant at the 0.05 probability level. The length of sub-apical compartments did not differ between the strains, averaging $76 \mu \mathrm{m}$, but variation in compartment length was significantly greater in the aps $A 6$ mutant (variance ratio $3 \cdot 14$ ).

Fig. 2 shows the mean frequency of nuclei per eyepiece unit length for the 16 hyphae of each genotype, aligned by their tips in Fig. 2(a), and by their first septa in Fig. 2(b). In both strains there is a gap at the very tip of the hypha, then the density of nuclei climbs steeply, followed by a gradual decline towards the rear of the compartment. However, the overall density of nuclei per unit was $51 \%$ greater in the mutant than in the wild-type. In contrast, the frequency of nuclei in compartments behind the tip was similar in the two strains: 1.09 and 1.14 nuclei per unit length for wild-type and mutant, respectively.

In both tips and subapical compartments the clustering of 

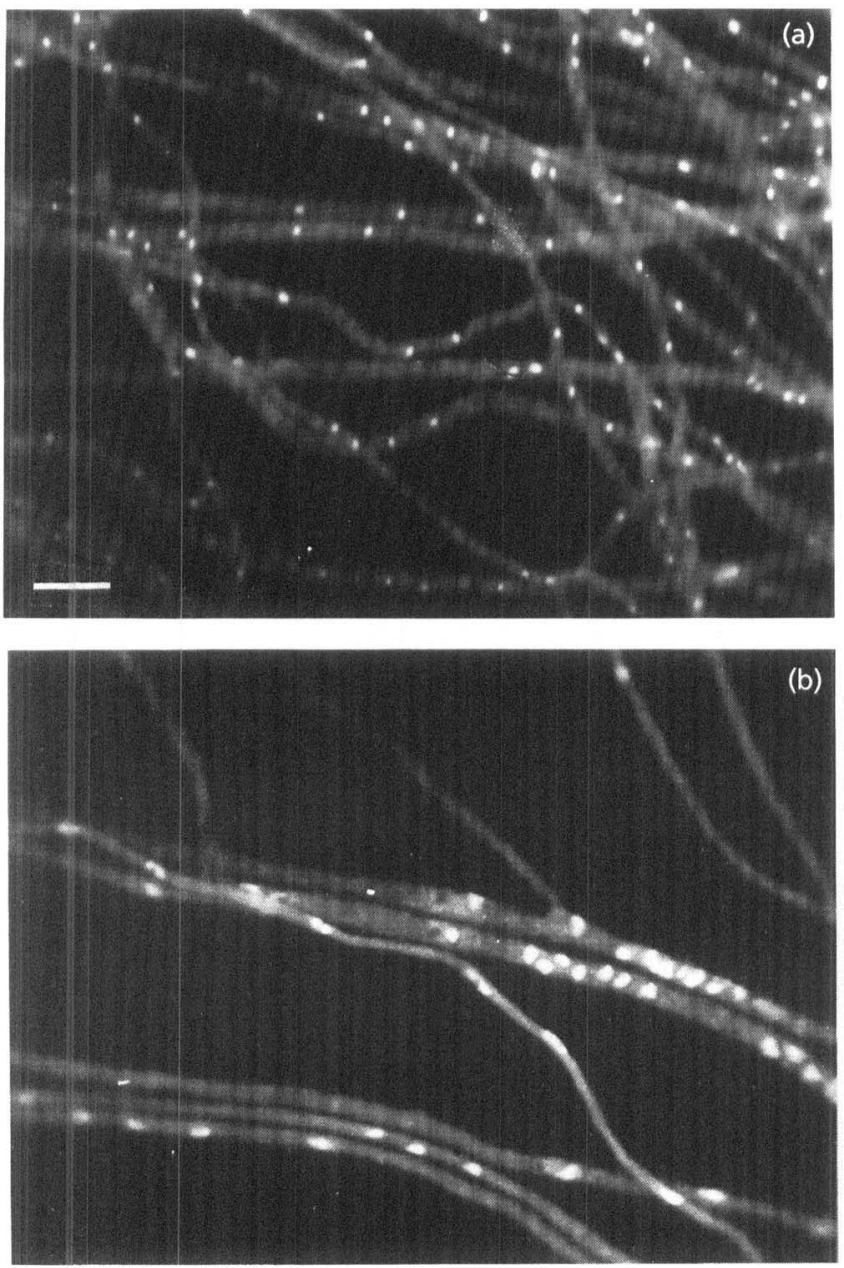

Fig. 3. Nuclear distribution in wild-type and aps $A 6$ hyphae. The mycelium was grown on slides and nuclei were visualized by acridine orange fluorescence. (a) Wild-type, (b) apsA6. Bar, $10 \mu \mathrm{m}$.

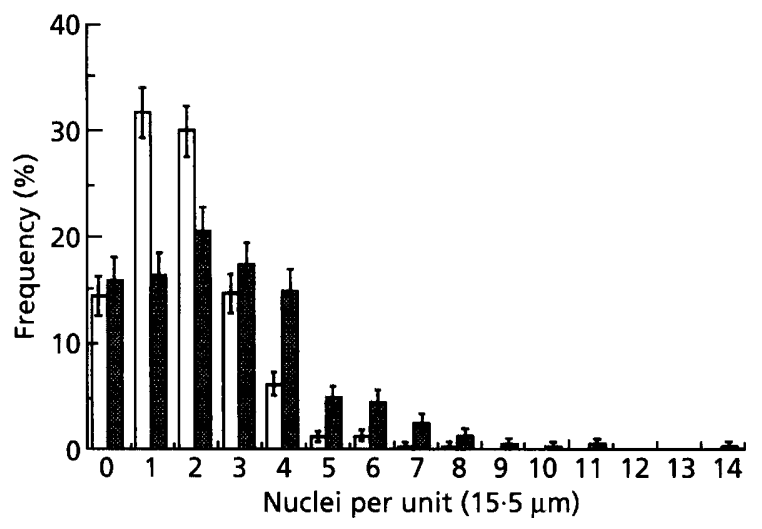

Fig. 4. Frequencies of units $(15 \cdot 5 \mu \mathrm{m})$ containing different numbers of nuclei in wild-type and apsA6 hyphal tip cells. Nuclei per eyepiece micrometer unit length $(15.5 \mu \mathrm{m})$ were counted in the tip cells of 16 hyphae of each type (see Fig. 2). Open bars, wild-type; shaded bars, apsA6. Error bars = SEM.

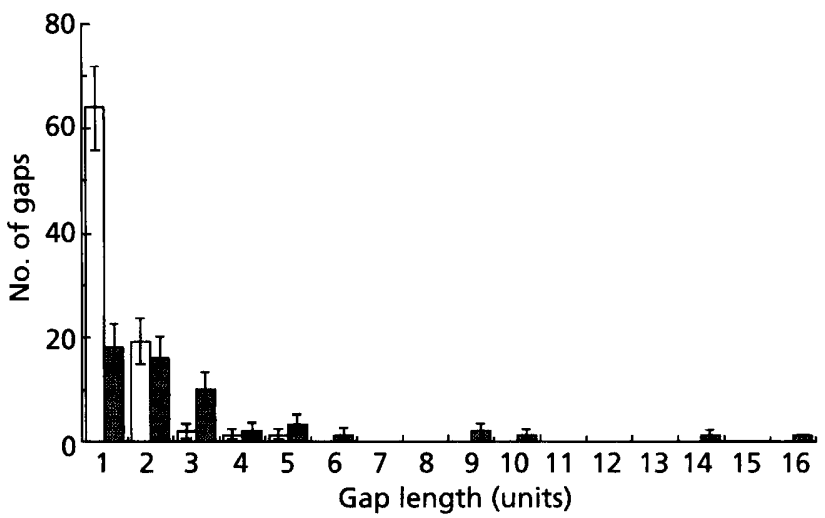

Fig. 5. Size and frequency of anucleate spaces of more than one unit length in wild-type and apsA6 hyphae. The unit length is $15.5 \mu \mathrm{m}$; gaps less than this length were ignored. Sixteen hyphae of each genotype were examined (see Fig. 2), for a distance extending 40 eyepiece micrometer units $(620 \mu \mathrm{m})$ back from each hyphal tip at the colony edge, and including both apical and sub-apical compartments. Open bars, wild-type; shaded bars, apsA6. The gap which is always present at the hyphal tip (see Fig. 2) was not included in the count. The total number of anucleate units was 117 in the wild-type hyphae and 167 in apsA6. Error bars = SEM.

nuclei was apparent to the eye (see Fig. 2). The irregular distribution of nuclei is investigated in Figs 4 and 5. Fig. 4 compares the frequency of nuclei per unit length in individual hyphal tips; in the wild-type, $63 \%$ of units contained two or three nuclei, whilst in the mutant there was a much wider spread of nuclear frequencies, one unit containing as many as 14 nuclei. The $\chi^{2}$ value for the difference between the two distributions was $69 \cdot 7$ (7 degrees of freedom) which is significant at the 0.001 probability level. In apical compartments of both strains the nuclear frequency was such that there were few gaps as long as the $15.5 \mu \mathrm{m}$ unit used, except at the very tip and rear of the compartment. However the subapical region of the aps $A 6$ mutant included many long compartments which appeared virtually empty. This is quantified in Fig. 5 which shows that in the 16 wild-type hyphae scanned (total length $9.92 \mathrm{~mm}$ ) there were only 53 intervals between adjacent nuclei that were longer than $15.5 \mu \mathrm{m}$, but in the same length of aps $A 6$ hyphae there were 149 such intervals, the longest gap being 16 units $(248 \mu \mathrm{m})$. The $2 \times 3 \chi^{2}$ value for gaps of 1,2 and $>2$ units length was 44.9 which is significant at the 0.001 probability level.

Oakley \& Morris $(1980,1981)$ have shown that nuclear migration in spore germlings is dependent on microtubules. As a possible indicator of a microtubule defect in aps mutants, they were screened for sensitivity to the microtubule inhibitor $p$-fluorophenylalanine. No difference from the wild-type was detected. A diploid strain homozygous for aps $A 6$ was also constructed and inspected for instability. Any mitotic instability is expected to be revealed in diploids by the formation of haploid sectors. No abnormal spontaneous instability of the homozygous aps $A$ diploid was seen. 


\section{Sexual phenotype}

Selfed cleistothecia of aps mutants were examined for ascospore production. aps $A 18$ was sexually sterile but all other aps. $A$ mutants appeared normal, both in ascospore numbers and appearance, and ascospore viability was estimated at $30 \%$, which was similar to that of wild-type controls. All aps $B$ mutants, in contrast, gave low yields of ascospores; many ascospores were oversized or misshapen, and a few appeared empty. Their viability was also very low, averaging $4 \cdot 2 \%$.

\section{DISCUSSION}

From a study of the wild-type, it is not immediately apparent that nuclear migration is an active process in fungi such as $A$. nidulans. Nuclei do not appear to mix actively in heterokaryons (Clutterbuck \& Roper, 1966), and since the fungus is homothallic, nuclear migration is not a necessity for mating. However, two sets of mutants deficient in nuclear movement have now been described. In addition to the aps mutants described here, Morris (1975) isolated a series of nud (nuclear distribution) mutants in which the nuclei fail to migrate out of the conidium on germination. The nud mutants are all temperature-conditional lethals.

Curiously enough, the properties of nud and aps mutants scarcely overlap, despite the fact that both show deficiencies in nuclear movement. The map locations of the two sets of loci are also different (Clutterbuck, 1903). As shown here, the aps defect is not confined to conidiation, but it might be specific to mature colonies, since no abnormality was seen in germlings. aps $B$, but not aps $A$ mutants are also affected in sexual reproduction, although, as in conidiation, the blockage is not absolute. While a condition of isolation of the nud mutants was that they were conditional lethals, aps mutants are only slightly defective in hyphal growth. It is possible that this is because all aps mutants isolated are leaky, and the slight variations in hyphal phenotype might be seen as support for this hypothesis. In contrast, the uniformity of the conidial phenotype and the fact that the lowest growth rate seen was $68 \%$ of the wild-type, suggests that the phenotype seen is that of the null mutant at these loci. This is a point that should be settled by disruption of the cloned genes.

The hyphal abnormality suggests that in the wild-type there exists a mechanism responsible for uniform distribution of nuclei. It is possible that this is essentially a dispersive mechanism that could also account for the migration of nuclei to the periphery of the conidiophore vesicle, such that one nucleus ends up in the mouth of each metula bud. Alternatively, there may be a more precise mechanism that directs nuclei to specified localities in the cell which might include branch sites and metula buds. It does not appear that nuclei are specifically directed to growing tips in general, since the tips of vegetative hyphae are devoid of nuclei (Fig. 2).

Functional microtubules are required for migration of nuclei, although not for movements of mitochondria (Oakley \& Rinehart, 1985). The evidence for this comes from the sensitivity of nuclear movement to the microtubule inhibitor benomyl (Oakley \& Morris, 1980), and also from failure of nuclear migration in the ben $A 33$ mutant, which encodes a defective $\beta$-tubulin (Oakley \& Morris, 1981). However, the microtubules are apparently unaffected in nudB and nudC mutants (Mayer et al., 1988; Osmani et al., 1990), and nuclear division, although not specifically studied here, appears to be normal in aps mutants. Admittedly, giant nuclei occasionally seen in the hyphae of aps mutants might suggest a mitotic defect in these mutants, but no support for such a defect was gained from mitotic inhibitor or diploid stability tests. An alternative explanation for giant nuclei would be that the abnormal distribution of nuclei in aps mutants allowed DNA replication by isolated nuclei which then failed to receive a signal to divide.

It is therefore apparent that the products of both nud and aps loci are required, in addition to functional microtubules, for nuclear movement. The $n u d C$ and nud $A$ genes have been cloned (Osmani et al., 1990, N. R. Morris and others, personal communication). The sequence of nud $A$ suggests that it encodes a dynein, but nud $C$ appears to encode a $22 \mathrm{kDa}$ protein of unknown function. Both aps genes have also been cloned (W. E. Timberlake, R. Fischer and L. Robertson, personal communication) and the sequence of aps $A$ has been determined: like nudC it does not appear to encode any recognizable cell motor component. It therefore remains a possibility that some of these genes, and the aps genes are good candidates for such a function, carry a responsibility for nuclear navigation rather than nuclear movement per se.

\section{REFERENCES}

Bailey, N. T. J. (1959). Statistical Methods in Biology. London: Hodder and Stoughton.

Bergen, L. G. \& Morris, N. R. (1983). Kinetics of the nuclear division cycle of Aspergillus nidulans. J Bacteriol 156, 155-160.

Clutterbuck, A. J. (1969a). A mutational analysis of conidial development in Aspergillus nidulans. Genetics 63, 317-327.

Clutterbuck, A. J. (1969b). Cell volume per nucleus in haploid and diploid strains of Aspergillus nidulans. J Gen Microbiol 55, 291299.

Clutterbuck, A. J. (1974). Aspergillus nidulans. In Handbook of Genetics, vol. 1. Bacteria, Bacteriophages and Fungi, pp. 447-510. Edited by R. C. King. New York: Plenum Press.

Clutterbuck, A. J. (1977). The genetics of conidiation in Aspergillus nidulans. In Genetics and Pbysiology of Aspergillus, pp. 305-317. Edited by J. E. Smith \& J. A. Pateman. London: Academic Press.

Clutterbuck, A. J. (1993). Aspergillus nidulans, nuclear genes. In Genetic Maps. Locus Maps of Complex Genomes, Gth edn, pp. 3.71-3.84. Edited by S. J. O'Brien. Cold Spring Harbor, NY: Cold Spring Harbor Laboratory.

Clutterbuck, A. J. \& Roper, J. A. (1966). A direct determination of nuclear distribution in heterokaryons of Aspergillus nidulans. Genet Res 7, 185-194.

Clutterbuck, A. J. \& Timberlake, W. E. (1992). (ienetic regulation of sporulation in the fungus Aspergillus nidulans. In Development, the Molecular Approach, pp. 103-120. Edited by V. E. A. Russo, S. Brody, D. Cove \& S. Ottolenghi. Berlin: Springer-Verlag.

Mayer, S. L. F., Kaminsky, G. W. \& Heath, I. B. (1988). Nuclear migration in a nud mutant of Aspergillus nidulans is inhibited in the 
presence of a quantitatively normal population of microtubules. $J$ Cell Biol 106, 773-778.

Mims, C. W., Richardson, E. A. \& Timberlake, W. E. (1988). Ultrastructural analysis of conidiophore development in the fungus Aspergillus nidulans using freeze-substitution. Protoplasma 144, 132-141.

Mooney, J. L. \& Yager, L. N. (1990). Light is required for conidiation in Aspergillus nidulans. Genes \& Dev 4, 1473-1482.

Morris, N. R. (1975). Mitotic mutants of Aspergillus nidulans. Genet Res 26, 237-254.

Oakley, B. R. \& Morris, N. R. (1980). Nuclear movement is $\beta$ tubulin-dependent in Aspergillus nidulans. Cell 19, 255-262.

Oakley, B. R. \& Morris, N. R. (1981). A $\beta$-tubulin mutation in Aspergillus nidulans that blocks microtubule function without blocking assembly. Cell 24, 837-845.
Oakley, B. R. \& Rinehart, J. E. (1985). Mitochondria and nuclei move by different mechanisms in Aspergillus nidulans. J Cell Biol 101, 2392-2397.

Osmani, A. H., Osmani, S. A. \& Morris, N. R. (1990). The molecular cloning and identification of a gene product specifically required for nuclear movement in Aspergillus nidulans. J Cell Biol 111, 543-551.

Pontecorvo, G., Roper, J. A., Hemmons, L. M., Macdonald, K. D. \& Bufton, A. W. J. (1953). The genetics of Aspergillus nidulans. Adv Genet 5, 141-238.

Timberlake, W. E. (1990). Molecular genetics of Aspergillus development. Annu Rev Genet 24, 5-36.

Received 27 September 1993; revised 29 November 1993; accepted 7 December 1993. 